\title{
A prognostic nomogram based on lymph node ratio for postoperative vulvar squamous cell carcinoma from the Surveillance, Epidemiology, and End Results database: a retrospective cohort study
}

\author{
Lei Lei ${ }^{1} \wedge$, Liao $\operatorname{Tan}^{2}$, Xingping Zhao ${ }^{1}$, Fei Zeng ${ }^{1}$, Dabao Xu ${ }^{1}$ \\ ${ }^{1}$ Department of Obstetrics and Gynecology, Third Xiangya Hospital, Central South University, Changsha, China; ${ }^{2}$ Department of Cardiovascular \\ Medicine, Xiangya Hospital, Central South University, Changsha, China \\ Contributions: (I) Conception and design: D Xu, F Zeng, L Lei; (II) Administrative support: D Xu, F Zeng; (III) Provision of study materials or \\ patients: None; (IV) Collection and assembly of data: L Lei, L Tan; (V) Data analysis and interpretation: L Tan, X Zhao; (VI) Manuscript writing: All \\ authors; (VII) Final approval of manuscript: All authors. \\ Correspondence to: Dabao Xu, MD. Department of Obstetrics and Gynecology, Third Xiangya Hospital of Central South University, 138 Tongzipo \\ Rd., Changsha 410013, China. Email: dabaoxu@yahoo.com; Fei Zeng, MD. Department of Obstetrics and Gynecology, Third Xiangya Hospital of \\ Central South University, 138 Tongzipo Rd., Changsha 410013, China. Email: zengfeilyx@163.com.
}

Background: Vulvar carcinoma is a rare gynecological malignancy. The most commonly used staging
system for vulvar cancer is the 2009 International Federation of Gynecology and Obstetrics (FIGO) staging
system. Nevertheless, it does not incorporate many indispensable prognostic parameters, which prominently
influence vulvar cancer patient survival. Thus, the development of a prediction model for evaluating survival
prognosis in postoperative vulvar squamous cell cancer patients is of vital importance.
Methods: Data from 2,166 patients with pathologically confirmed diagnosis of vulvar squamous cell
carcinoma from 2004 to 2015 were acquired from the Surveillance, Epidemiology, and End Results (SEER)
database. Thirty percent of the patients were randomly assigned to the validation group, and the remainder
were used to develop the nomogram. Parameters that significantly correlated with overall survival (OS) were
used to create the nomogram. Concordance index (C-index), calibration curve, and decision curve analysis
(DCA) were used to assess the predictive accuracy and discriminability of the nomogram model. Additionally,
the C-index and DCA of the nomogram and the FIGO staging system were compared.
Results: Following multivariate analysis of the training cohort, independent factors for OS, including race,
age at diagnosis, marital status, FIGO stage, tumor diameter, and lymph node ratio (LNR), were included in
the nomogram model. The calibration curve indicated a high correlation between the nomogram-predicted
and observed survival probability. The C-index of the nomogram in the training cohort was 0.772 (95\% CI:
0.752-0.792), statistically superior to the C-index value of the FIGO staging system (0.676, 95\% CI: $0.654-$
0.698). In DCA, compared to the FIGO staging system, this nomogram showed a greater net benefit and a
wider range of threshold probability. Results were verified by an internal validation cohort.
Conclusions: Our nomogram, based on LNR, showed superior prognostic predictive accuracy compared
with the FIGO staging system for predicting OS in postoperative vulvar squamous cell carcinoma patients.

Keywords: Lymph node ratio (LNR); prognostic nomogram; vulvar cancer

Submitted Apr 10, 2020. Accepted for publication Sep 04, 2020.

doi: $10.21037 / \mathrm{atm}-20-3240$

View this article at: http://dx.doi.org/10.21037/atm-20-3240

^ ORCID: 0000-0002-4291-3532. 


\section{Introduction}

Vulvar carcinoma is a rare malignant gynecologic tumor that accounts for $4-5 \%$ of all gynecological malignancies in the United States, with an estimated incidence of 6,190 new cases and 1,200 deaths in 2018 alone (1). Vulvar squamous cell carcinoma, the most common pathological subtype, is generally thought to occur in postmenopausal women. However, in recent years, due to the increase in human papillomavirus (HPV) infections worldwide, the average age of onset has decreased (2). Generally, lymph node (LN) involvement is considered a significant factor in the therapeutic decision-making and prognosis of vulvar carcinoma (3). Radical vulvectomy with complete inguinofemoral lymphadenectomy remains the standard approach for the management of vulvar cancer in lymph node-positive patients. The number of positive lymph nodes (PLNs) was included in the 2009 International Federation of Gynecology and Obstetrics (FIGO) staging system (4). The five-year overall survival (OS) rate ranges from $30 \%$ to $58.5 \%$ for lymph node-positive patients, whereas it ranges from $64.7 \%$ to $90.9 \%$ in lymph node-negative patients (5-7). The OS of LN-positive patients varies greatly depending on the extent of LN involvement. However, clinicians demonstrated that the number of PLNs might not adequately reflect $\mathrm{LN}$ involvement in clinical practice, because the degree of intraoperative $\mathrm{LN}$ evaluation influences the number of involved resected lymph nodes (RLNs) and the detection rate of LN metastases. Therefore, if surgical detection of $\mathrm{LN}$ is limited in LN-positive patients, it is possible that cases of $\mathrm{LN}$ metastasis could be overlooked.

The lymph node ratio (LNR) is defined as the ratio of the number of PLNs to the total number of RLNs, which reflects not only the burden of lymph node metastasis but also the degree of operative LN evaluation. The LNR has emerged as a vital independent prognostic factor for survival outcomes, and is superior to evaluation based on LN status in solid tumor malignancies, including cervical (8), endometrial (9), ovarian (10), breast (11), esophageal (12), gastric (13), and colorectal cancers (14). In two retrospective multicenter cohort trial analyses of the VULCAN (15) and the AGO CaRE-1 trial datasets (16), LNR appeared to be a consistent, independent prognostic parameter in vulvar cancer patients, and outperformed the number of PLNs in survival analyses.

The most commonly used staging system for vulvar cancer is the FIGO score staging system. Nevertheless, studies have shown that the FIGO staging system does not incorporate many indispensable prognostic parameters, which can greatly influence vulvar squamous cell cancer patient survival, such as patient characteristics, tumor cell differentiation, LNR, HPV status, and additional factors relevant to molecular pathways of prognosis (17-21). Thus, it is vital to develop a prediction model for evaluating survival prognosis in vulvar squamous cell cancer patients that incorporates tumor status as well as patient characteristics.

Nomogram-based statistical methods are intuitive statistical tools that quantify risk. They have advantages over traditional staging systems for many carcinomas, and have been generally implemented in oncologic prognosisassociated clinical practices $(22,23)$. Here, we developed a nomogram using LNR, based on the assumption that this is a vital prognostic parameter and is superior to PLNs in survival prediction for vulvar squamous cell carcinoma patients. Furthermore, this nomogram may show superior prognostic predictive accuracy compared with the current FIGO staging system.

We present the following article in accordance with STROBE guideline checklist (available at http://dx.doi. org/10.21037/atm-20-3240).

\section{Methods}

\section{Data source and collection}

In this retrospective cohort study, patients diagnosed with vulvar cancer between 2004 and 2015 were included from the Surveillance, Epidemiology, and End Results (SEER) database. Methods of data collection and follow-up of patients are available on the SEER database. Inclusion criteria were as follows: histopathologically proven vulvar squamous cell carcinoma; and surgical treatment included lymphadenectomy. Exclusion criteria were: a history of other malignancies; a history of neoadjuvant anticancer therapy or sentinel lymph node biopsy; unexplained mortality; and unknown variables, such as race/ethnicity, age of diagnosis, tumor grade, FIGO stage, tumor diameter, marital status, number of PLNs, and number of RLNs.

Based on the aforementioned criteria, a total of 2,166 vulvar squamous cell carcinoma patients were included in this study. Thirty percent of the patients were randomly assigned to the validation group, and the remainder were used to develop the nomograms (training cohort, $\mathrm{N}=1,518$; validation cohort, $\mathrm{N}=648$ ). The flow chart for detailed patient selection is presented in Figure 1. 


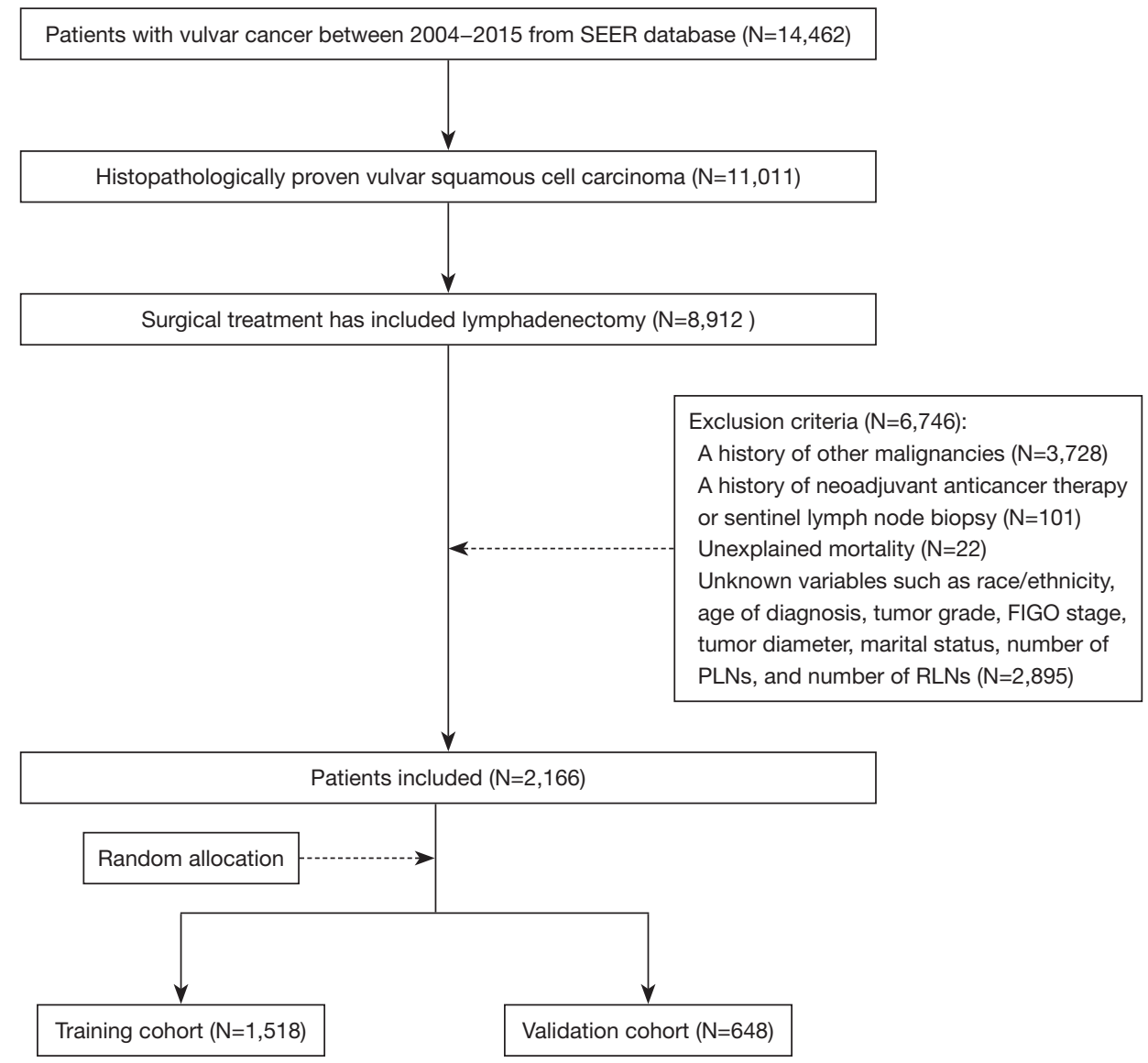

Figure 1 Flow chart of patient selection. FIGO, International Federation of Gynecology and Obstetrics; LNR, lymph node ratio; PLNs, number of positive lymph nodes; RLNs, number of resected lymph nodes.

\section{Prognostic variables}

The following clinical variables were extracted: race/ ethnicity (black, white, or others); age at diagnosis $(<50$, $50-64$, or $>64$ years); tumor grade (I, well differentiated; II, moderately differentiated; or III, poorly differentiated/ undifferentiated); tumor diameter $(<4$, or $\geq 4 \mathrm{~cm})$; FIGO stage (I, II, III or IV); and marital status (single, married, divorced, widowed, or others). The number of PLNs was subdivided into four groups: zero, one, two, and three or more. The LNR was stratified into the following three risk groups, according to LNR as previously published in the report from Gynecologic Oncology Group (GOG) protocol \#37 (24): $0.0,0.0-0.2$, and $>0.2$. The outcome of this study was OS; overall survival was defined as the interval from the surgical date to date of death from any cause or last follow-up.

\section{Statistical analysis}

Count and percentage were used to describe categorical measurements, whereas mean and range were used to present continuous measurements. The chi-square or Fisher's exact test was used to compare the categorical measurements, while $t$ test or Mann-Whitney $\mathrm{U}$ test was used to compare continuous variables with an abnormal distribution. The Kaplan-Meier method was used to depict survival curves. Cox regression analysis was conducted for multivariate analyses. A nomogram was formulated with potential risk factors $(\mathrm{P}<0.05)$ based on results from the multivariate analysis. The concordance index (C-index), calibration curve, and decision curve analysis (DCA) were calculated to evaluate the predictive performance. DCA was performed to test the clinical usefulness of the model. The predictive performance was validated using an independent 
internal cohort. Additionally, the predictive ability of the nomogram was compared with the FIGO staging system by calculating the C-index and constructing the DCA model. A larger C-index and wider range of threshold probability indicated a superior prognostic prediction. All statistical analyses and graph construction were performed using $\mathrm{R}$ (version 3.5.1; www.r-project.org). $\mathrm{P}$ value $<0.05$ was defined as statistically significant.

\section{Results}

\section{Patient characteristics}

Overall, 2,166 postoperative patients diagnosed with vulvar squamous cell carcinoma between 2004 and 2015 from the SEER database were included. The main clinical characteristics and baseline demographic of the study subjects are summarized in Table 1.

\section{Independent Prognostic Factor for OS}

Univariate and multivariate analyses were performed to identify factors that were significantly correlated with OS (Table 2). In the training cohort, race, age at diagnosis, marital status, tumor grade, FIGO stage, tumor diameter, and LNR were significantly associated with OS by Univariate analysis $(\mathrm{P}<0.05)$. Further multivariate analysis indicated that race, age at diagnosis, marital status, FIGO stage, tumor diameter, and LNR were independent risk factors for $\mathrm{OS}(\mathrm{P}<0.05)$.

Survival analysis of Kaplan-Meier curves demonstrated a significant correlation between poorer OS and the group with an LNR greater than 0.2 compared to groups with lower LNR, in both the training and validation cohorts (Figure 2).

\section{Construction and validation of the Prognostic Nomogram}

Based on the multivariate analysis results, the prognostic nomogram was formulated to predict the 3 - and 5 -year OS in the training cohort (Figure 3). In the training cohort, a C-index of 0.772 (95\% CI: 0.752-0.792) was observed for the nomogram. For the validation cohort, the C-index was 0.784 (95\% CI: $0.75-0.813$ ). Calibration plots for the OS nomogram validation indicated a high correlation between the predicted and observed probabilities of survival at 3- or 5 -year post-surgery for both the training (Figure $4 A, B$ ) and validation cohorts (Figure 4C,D).

\section{Comparison with the FIGO staging system}

In the training cohort, the $\mathrm{C}$-index of the nomogram cohort was 0.772 (95\% CI: 0.752-0.792), statistically higher than that of the FIGO staging system $(0.676,95 \%$ CI: $0.654-0.698)$. The C-index of our model $(0.784,95 \% \mathrm{CI}$ : $0.755-0.813)$ remained superior to that of the FIGO staging system $(0.693,95 \% \mathrm{CI}: 0.660-0.726)$ in the validation cohort.

In DCA, our nomogram presented a greater net benefit as well as a wider field of threshold probability compared to the FIGO staging system, both in the training cohort (Figure $5 A, B)$ and the validation cohort (Figure $5 C, D$ ), indicating that our model has superior predictive ability in predicting OS at 3- or 5-year. A higher threshold probability results in a more robust estimation of decision results. The results indicated that our formulated nomogram is a better predictive method for survival of patients with squamous cell vulvar cancer.

\section{Conclusions}

The FIGO score staging system is commonly used for vulvar cancer. However, controversies remain regarding the FIGO system, as a large number of additional risk factors have proven to be prognostic parameters for vulvar cancer patients. Consequently, the FIGO staging system appears to have lost its effectiveness in evaluating the prognosis of vulvar cancer. Moreover, the FIGO staging system was not specifically developed for postoperative prognostic prediction in squamous cell vulvar cancer patients, the most common type of vulvar cancer; thus, the predictive prognostic accuracy for these patients who then undergo surgery may be affected.

A nomogram is a quantitative hierarchical prediction model that has shown robust ability in survival prediction in many carcinomas $(25,26)$. Therefore, as the predominant treatment of vulvar carcinoma, specifically squamous cell carcinoma, is surgery, we constructed a prognostic nomogram for postoperative vulvar squamous cell cancer patients.

In this study, we assessed 2,166 patients with postoperative vulvar squamous cell cancer. In the univariate and multivariate analyses, LNR was considered as an independent predictive factor for prognosis, as was race, age at diagnosis, marital status, FIGO staging system, and tumor diameter. Finally, a nomogram based on these factors was formulated. The nomogram had advantages in survival prediction, and the 
Table 1 Demographic and clinicopathologic characteristics of patients with squamous cell carcinoma of vulva diagnosed between 2004 and 2015 from SEER database

\begin{tabular}{|c|c|c|c|c|c|c|}
\hline Variables & \multicolumn{2}{|c|}{ All $(\mathrm{N}=2,166)$} & \multicolumn{2}{|c|}{ Training cohort $(\mathrm{N}=1,518)$} & \multicolumn{2}{|c|}{ Validation cohort $(\mathrm{N}=648)$} \\
\hline \multicolumn{7}{|c|}{ Age of diagnosis (year) } \\
\hline$<50$ & 385 & 17.77 & 271 & 17.85 & 114 & 17.59 \\
\hline $50-64$ & 660 & 30.47 & 476 & 31.36 & 184 & 28.40 \\
\hline \multicolumn{7}{|l|}{ Race } \\
\hline Black & 162 & 7.48 & 114 & 7.51 & 48 & 7.41 \\
\hline White & 1930 & 89.10 & 1359 & 89.53 & 571 & 88.12 \\
\hline Others & 74 & 3.42 & 45 & 2.96 & 29 & 4.48 \\
\hline Divorced & 268 & 12.37 & 189 & 12.45 & 79 & 12.19 \\
\hline Widowed & 523 & 24.15 & 367 & 24.18 & 156 & 24.07 \\
\hline Others & 33 & 1.52 & 21 & 1.38 & 12 & 1.85 \\
\hline \multicolumn{7}{|l|}{ Tumor grade } \\
\hline I & 547 & 25.29 & 391 & 25.81 & 156 & 24.07 \\
\hline II & 1169 & 54.05 & 822 & 54.26 & 347 & 53.55 \\
\hline III & 447 & 20.67 & 302 & 19.93 & 145 & 22.38 \\
\hline II & 310 & 14.31 & 228 & 15.02 & 82 & 12.65 \\
\hline III & 729 & 33.66 & 489 & 32.21 & 240 & 37.04 \\
\hline IV & 42 & 1.94 & 21 & 1.38 & 21 & 3.24 \\
\hline \multicolumn{7}{|l|}{ LNR } \\
\hline 0 & 1404 & 63.64 & 1015 & 65.15 & 389 & 60.03 \\
\hline $0-0.2$ & 405 & 18.36 & 277 & 17.78 & 128 & 19.75 \\
\hline$>0.2$ & 397 & 18.00 & 266 & 17.07 & 131 & 20.22 \\
\hline \multicolumn{7}{|l|}{ PLN } \\
\hline Mean (range) & 0.87 & $0-40$ & 0.77 & $0-40$ & 1.1 & $0-23$ \\
\hline \multicolumn{7}{|l|}{ RLNs } \\
\hline Mean (range) & 10.20 & $1-66$ & 10.22 & $1-66$ & 10.14 & $1-43$ \\
\hline
\end{tabular}

FIGO, International Federation of Gynecology and Obstetrics; LNR, lymph node ratio; PLNs, number of positive lymph nodes; RLNs, number of resected lymph nodes. 
Table 2 Univariate and multivariate analyses of prognostic factors associated with overall survival of patients with squamous cell carcinoma of vulva in the training cohort

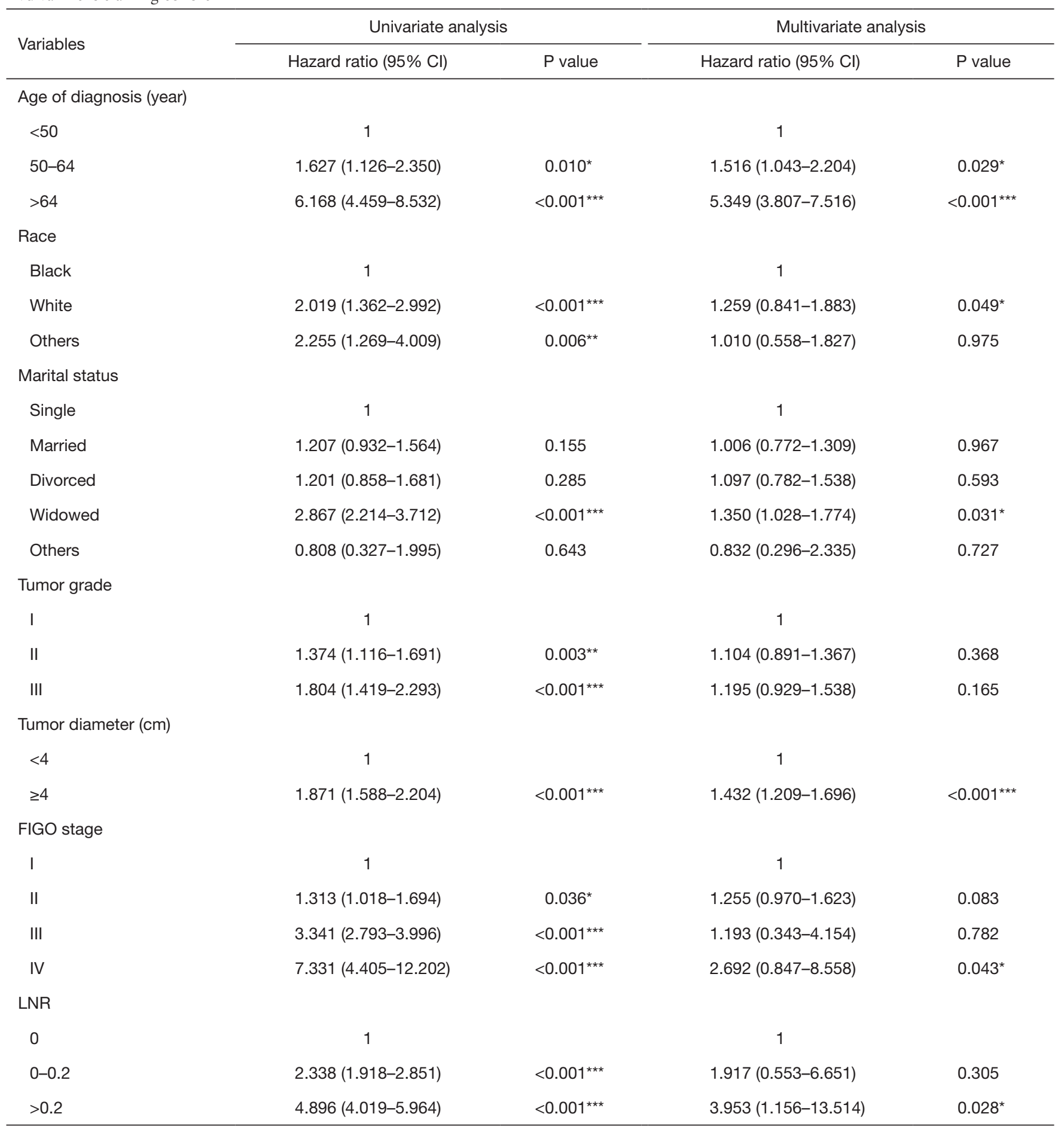

\footnotetext{
${ }^{*}, \mathrm{P}<0.05 ;{ }^{* *}, \mathrm{P}<0.01$; ${ }^{* \star}, \mathrm{P}<0.001$. FIGO, International Federation of Gynecology and Obstetrics; LNR, lymph node ratio.
} 
A

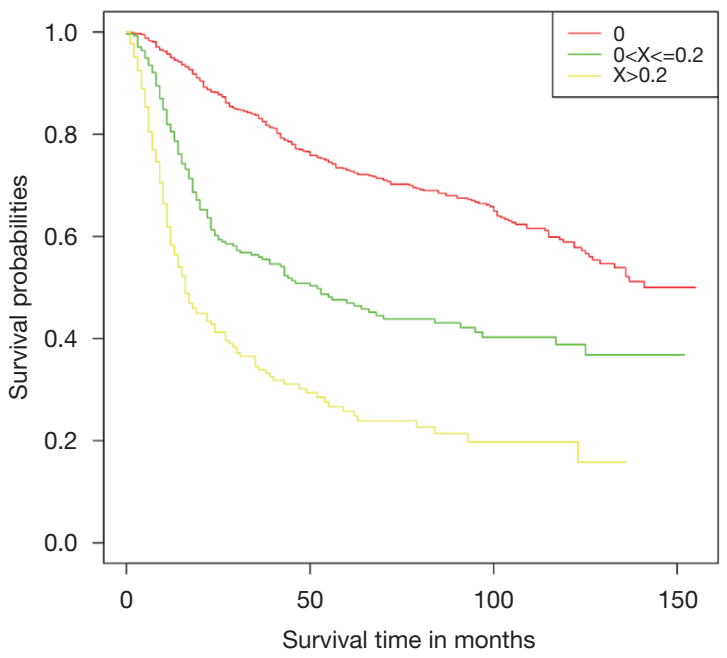

B

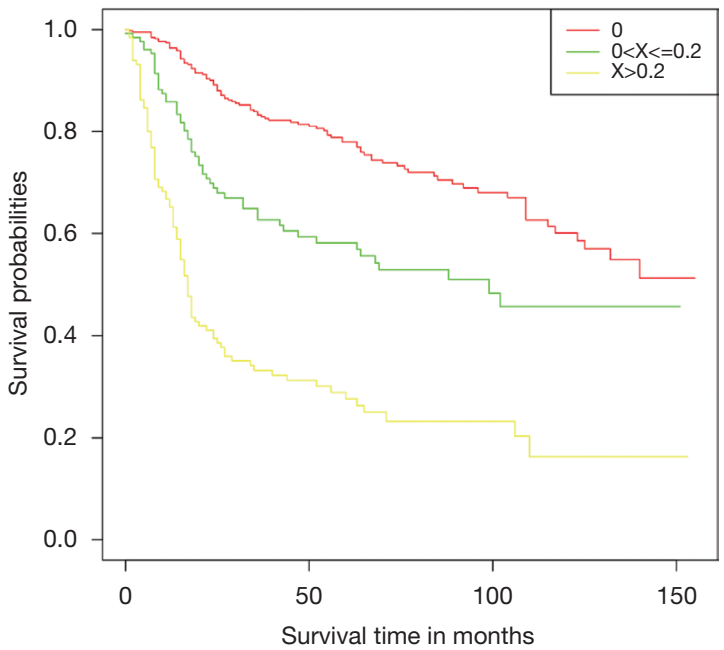

Figure 2 Kaplan-Meier survival curves for patients with vulvar squamous cell carcinoma in the training cohort (A) and validation cohort (B) according to LNR. LNR, lymph node ratio.

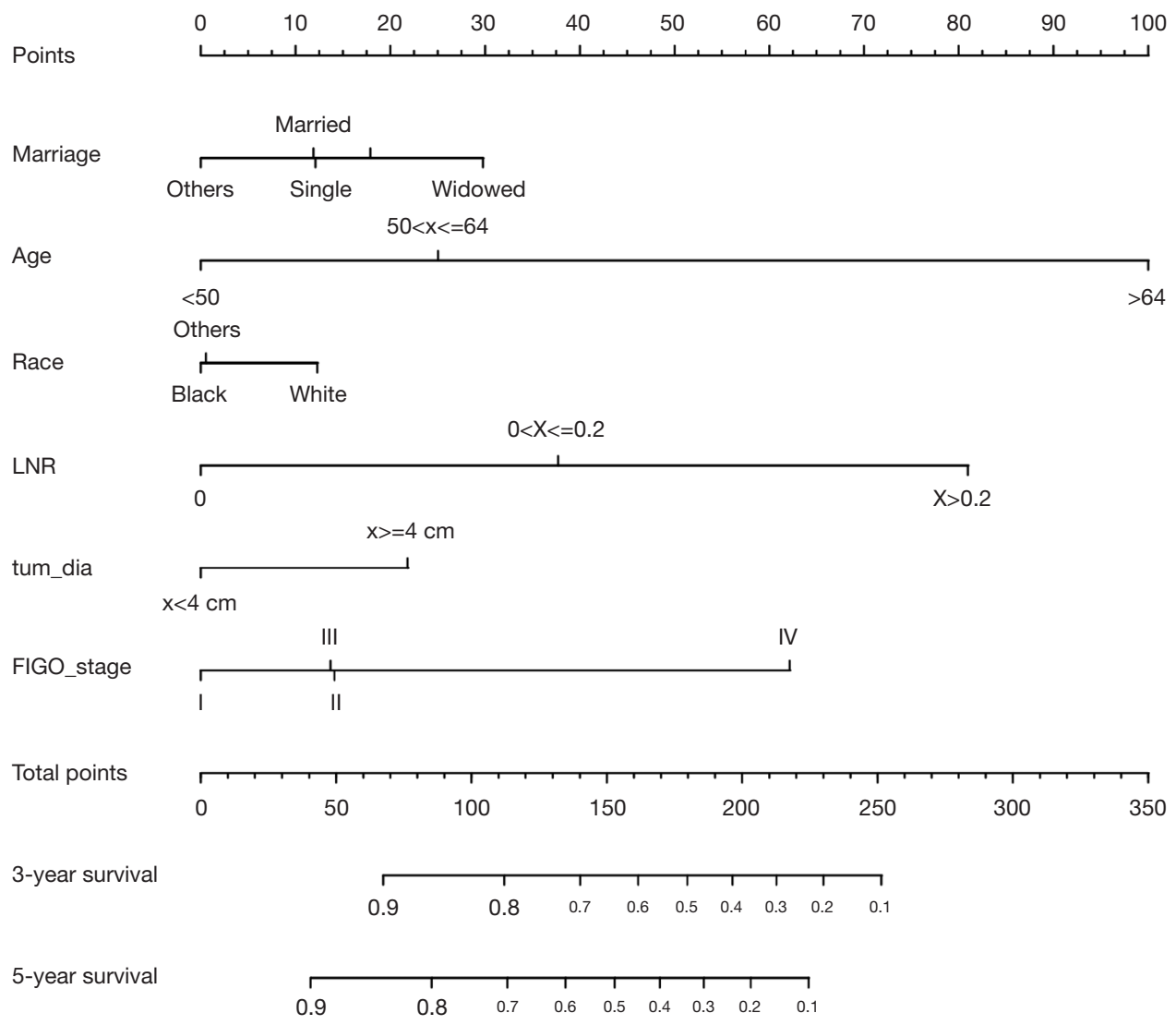

Figure 3 Overall survival-predicting nomogram for postoperative vulvar squamous cell carcinoma. LNR, lymph node ratio; tum_dia, tumor diameter; FIGO, International Federation of Gynecology and Obstetrics. 
A

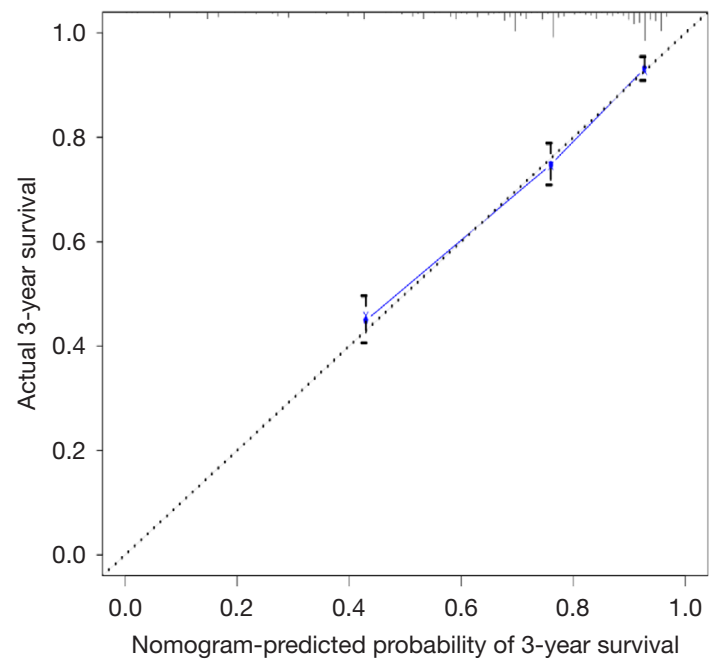

C

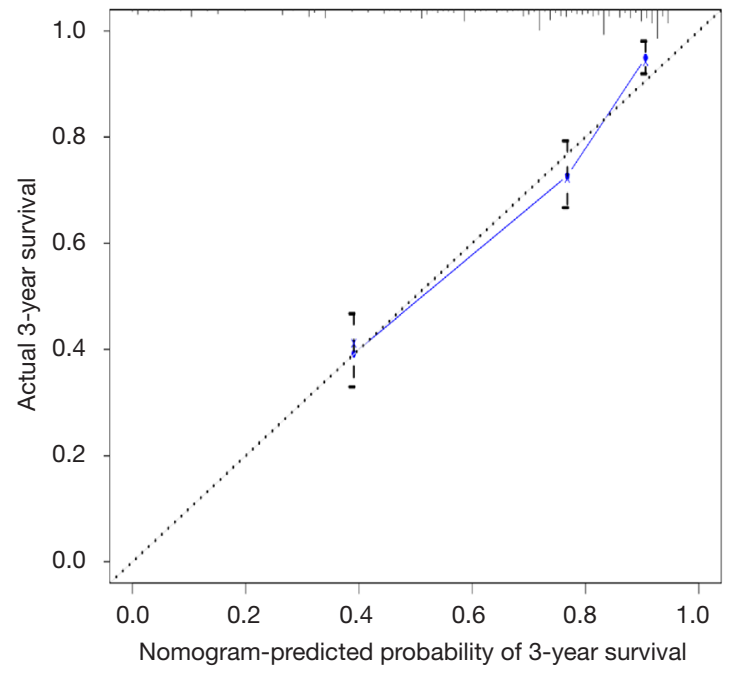

B

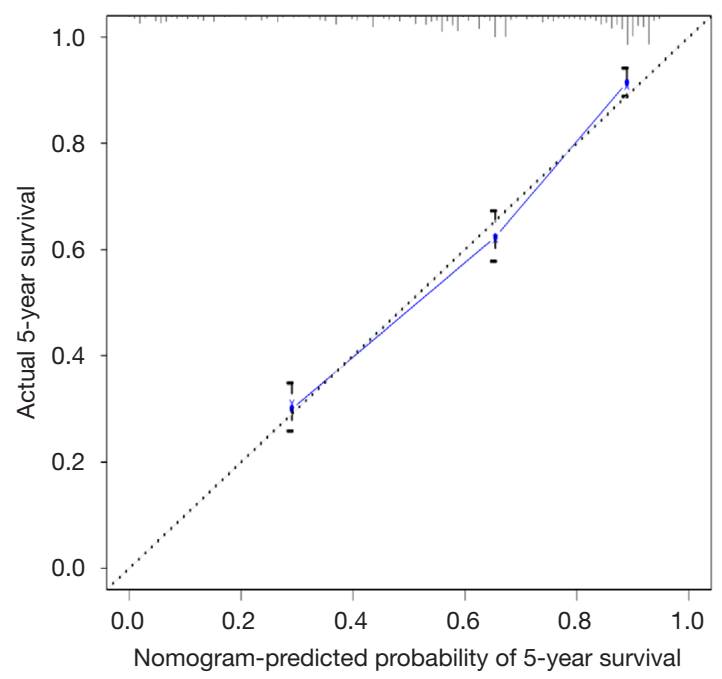

D

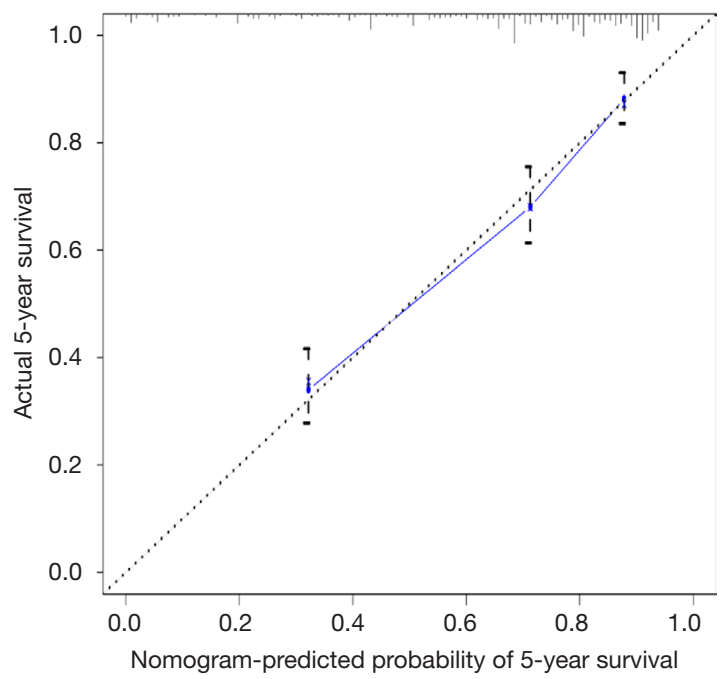

Figure 4 The calibration curve of the nomogram for predicting overall survival at 3- (A) and 5-year (B) in the training cohort, and at 3(C) and 5-year (D) in the validation cohort. The nomogram-predicted probability of overall survival is plotted on the $\mathrm{x}$-axis and the actual probability is on the $y$-axis.

C-index (0.772 and 0.784 for the training and validation cohorts, respectively), and the calibration curve, and DCA supported its predictive performance. Compared with the FIGO staging system, the nomogram demonstrated advanced efficiency for prognostic prediction.

In contrast to the FIGO staging system, tumor diameter and lymph node status remained in this model, and race, age at diagnosis, and marital status were further included in our formulated nomogram. Lymph node status is perceived to be one of the most significant factors for predicting OS and progression-free survival (PFS) in vulvar cancer patients $(3,27)$. However, the lymph node status of the FIGO staging system failed to adequately reflect the extent of disease, due to the influence of surgical operation, and the number of examined PLNs remained defective as a crucial predictive factor. Kunos et al. (24) first described LNR for prognostic assessment in patients with vulvar cancer. Patients with an LNR value $>0.2$ had an increased likelihood of contralateral 
A

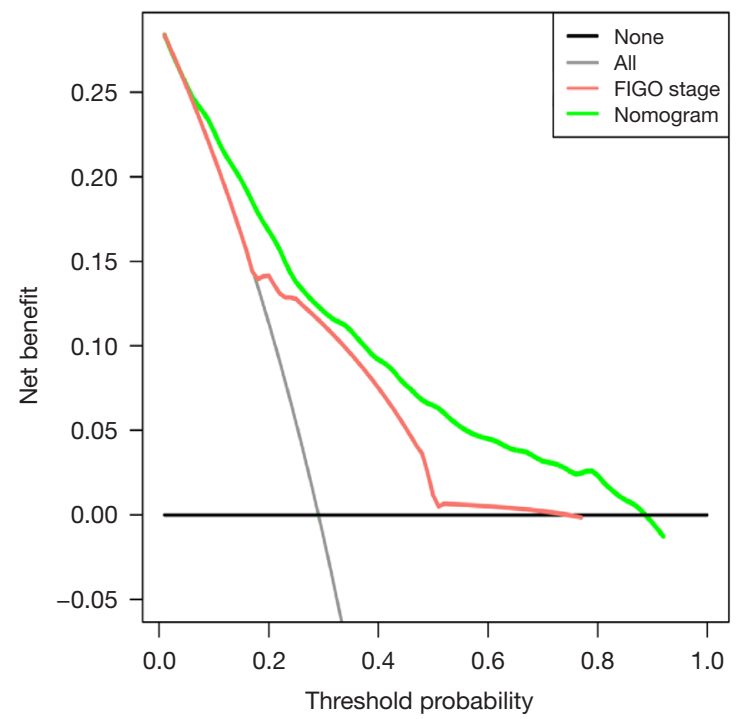

C

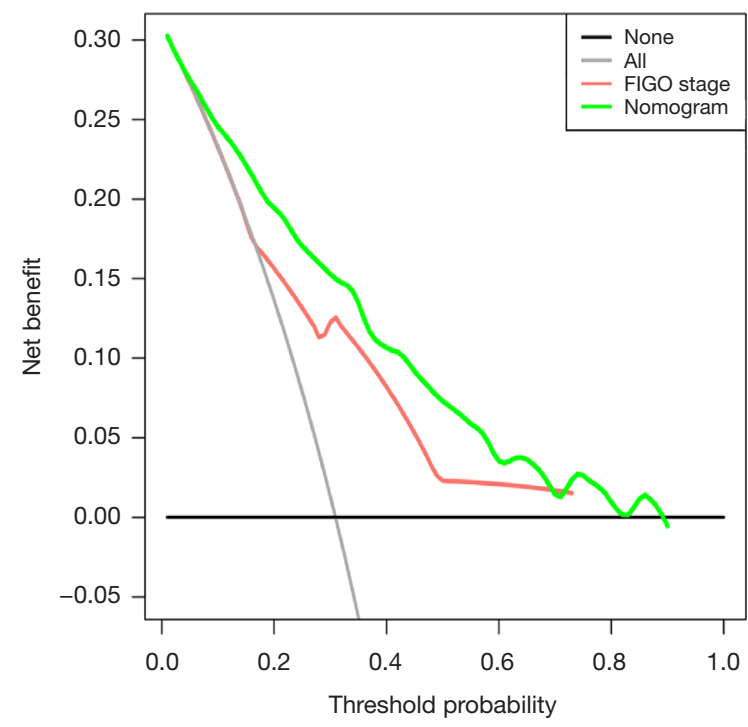

B

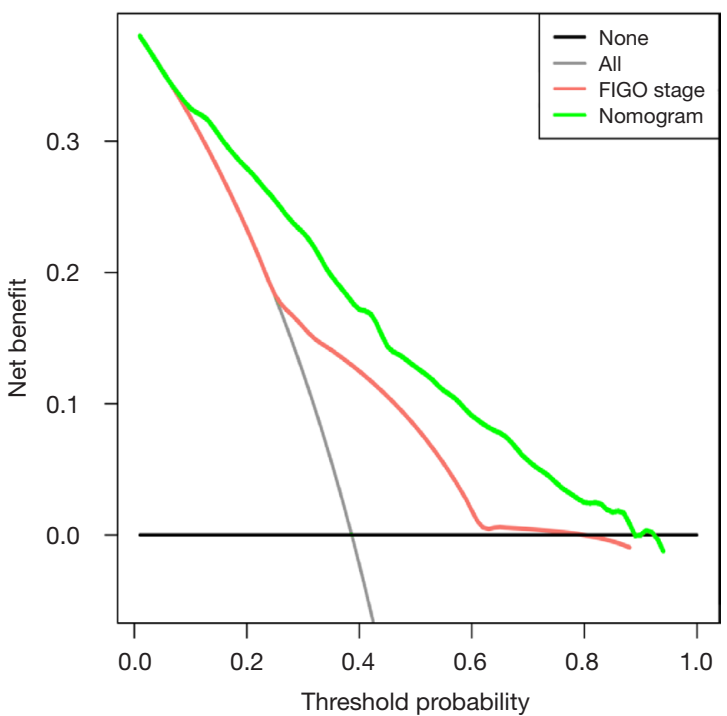

D

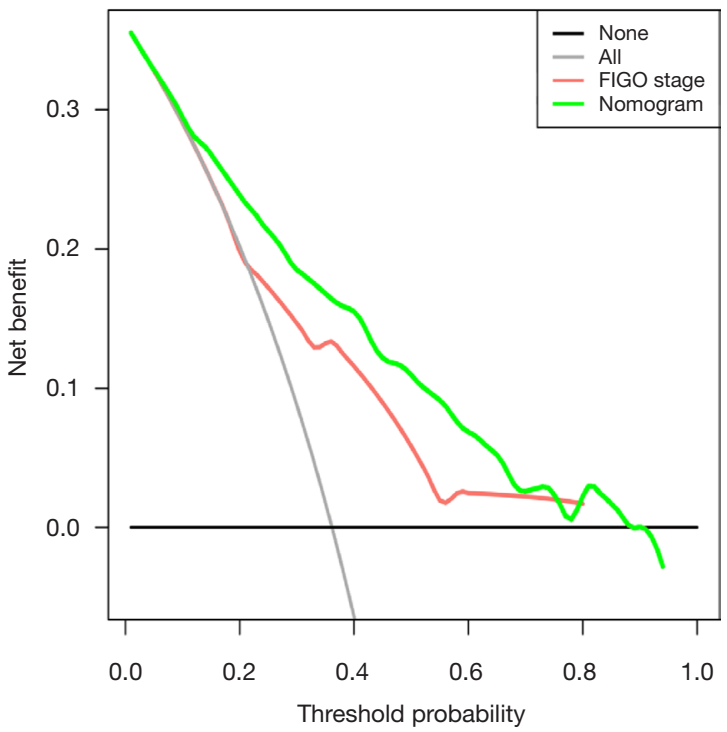

Figure 5 Decision curve analysis for postoperative vulvar squamous cell carcinoma using nomogram and FIGO staging system in terms of the 3- (A) and 5-year (B) overall survival in the training cohort, and the 3- (C) and 5-year (D) overall survival in the validation cohort. The $\mathrm{x}$-axis represents the threshold probability. The y-axis measures the net benefit. The threshold probability is where the expected benefit of treatment balances the expected benefit of avoiding treatment. The nomograms resulted in a superior net benefit to the FIGO staging system, with a wide range of threshold probabilities. FIGO, International Federation of Gynecology and Obstetrics.

PLNs, recurrence, and cancer-specific death compared with patients with an LNR value $\leq 0.2$. The prognostic significance of LNR was confirmed through a study of 1,047 patients who underwent surgery, including inguinal lymph node resection for vulvar squamous cell cancer, from a multicenter retrospective trial (the AGO CaRE-1 trial) (16). Our results suggest that a high LNR was relevant to poor OS, and agreed with the AGO CaRE-1 trial results. 
From a clinical point of view, these findings are persuasive, as LNR reflects not only the nodal disease burden, but also the quality and extent of lymphadenectomy.

In addition, previous studies demonstrated that age is a vital prognostic factor (28). Our multivariate analyses revealed that older age was an independent risk parameter, indicating that senior patients had poorer overall survival. This study also revealed that widowed patients had poorer outcomes than non-widowed patients, which concurs with a study showing an increased risk of cancer mortality in widows with vulvar cancer (19). Widowed cancer patients may suffer greater depression, anxiety, and distress than their non-widowed counterparts, and are less likely to receive surgical treatment and chemotherapy, potentially contributing to poorer cancer control. These findings indicate a need for social support and personalized care among widowed patients. Additionally, the important role of race in relation to survival was emphasized in previous analyses. Rauh-Hain et al. (18) reported that AfricanAmerican patients had improved survival compared to white patients, consistent with our results.

The most common staging system for vulvar carcinoma is the FIGO staging system; thus, we compared the prognostic nomogram and the FIGO staging system via the C-index and DCA. We discovered that the C-index of our nomogram for predicting OS in the training cohort was 0.772 (95\% CI: $0.752-0.792$ ), which was statistically superior to that of the FIGO staging system $(0.676,95 \%$ CI: 0.654-0.698), as was the result in the validation cohort. However, high prediction accuracy does not guarantee preferable clinical application (29). Therefore, in the present study, we used DCA to demonstrate that the nomogram obtained wider ranges of DCA and yielded better clinical validity and applicability.

In this study, the nomogram, consisting of six easily accessible variables, could conveniently provide a precise estimation of the individual survival rates to healthcare providers and patients. Additionally, it could facilitate the choice of postoperative treatment decision-making, such as adjuvant radiation, chemotherapy, or target-directed therapy. Moreover, this nomogram could assist in the formulation and adjustments of follow-up intervals for the purpose of achieving individualized disease monitoring. Another potential use of the nomogram could be to identify patients at high risk of death, allowing them to participate in clinical trials evaluating novel treatments for patients with an extremely poor prognosis.

While the current study makes sense in clinical practice, there are limitations that should be acknowledged. First, there is an inevitable selection bias due to using retrospective data from the SEER database, where the ethnic homogeneity of the patient population was a methodological limitation of our study. In addition, some prognostic factors, such as surgical tumor-free margin, serum tumor markers, and vascular infiltration, which may influence prognosis of postoperative vulvar cancer patients, are not available in the SEER database. Finally, although the prognostic nomogram model performed well in the current study, further validation is required in additional cohorts to enhance reliability. However, conducting a multicenter, large-scale prospective clinical trial of this rare disease remains a major challenge.

The formulated prognostic nomogram accurately and objectively predicted OS of vulvar squamous cell carcinoma. This may have a superior prognostic capability for patients with postoperative vulvar squamous cell carcinoma compared to the currently utilized FIGO staging system. Further studies to validate and improve this model are warranted.

\section{Acknowledgments}

Funding: This study is supported by Natural Science Foundation of China (Grant No. 81671492) and the Hunan Science and Technology Department (Grant No. 2018SK2102).

\section{Footnote}

Reporting Checklist: The authors have completed the STROBE guideline checklist. Available at http://dx.doi. org/10.21037/atm-20-3240

Conflicts of Interest: All authors have completed the ICMJE uniform disclosure form (available at http://dx.doi. org/10.21037/atm-20-3240). The authors have no conflicts of interest to declare.

Ethical Statement: The authors are accountable for all aspects of the work, ensuring that questions related to the accuracy or integrity of any part of the work are appropriately investigated and resolved.

Open Access Statement: This is an Open Access article distributed in accordance with the Creative Commons Attribution-NonCommercial-NoDerivs 4.0 International 
License (CC BY-NC-ND 4.0), which permits the noncommercial replication and distribution of the article with the strict proviso that no changes or edits are made and the original work is properly cited (including links to both the formal publication through the relevant DOI and the license). See: https://creativecommons.org/licenses/by-nc-nd/4.0/.

\section{References}

1. Siegel RL, Miller KD, Jemal A. Cancer statistics, 2018. CA Cancer J Clin 2018;68:7-30.

2. Kang YJ, Smith M, Barlow E, et al. Vulvar cancer in highincome countries: Increasing burden of disease. Int $\mathrm{J}$ Cancer 2017;141:2174-86.

3. Burger MP, Hollema H, Emanuels AG, et al. The importance of the groin node status for the survival of $\mathrm{T} 1$ and T2 vulvar carcinoma patients. Gynecol Oncol 1995;57:327-34.

4. Pecorelli S. Revised FIGO staging for carcinoma of the vulva, cervix, and endometrium. Int J Gynaecol Obstet 2009;105:103-4.

5. Baiocchi G, Silva CF, Rocha RM, et al. Prognostic value of the number and laterality of metastatic inguinal lymph nodes in vulvar cancer: revisiting the FIGO staging system. Eur J Surg Oncol 2013;39:780-5.

6. van der Steen S, de Nieuwenhof HP, Massuger L, et al. New FIGO staging system of vulvar cancer indeed provides a better reflection of prognosis. Gynecol Oncol 2010;119:520-5.

7. Zhou J, Shan G. The prognostic role of FIGO stage in patients with vulvar cancer: a systematic review and metaanalysis. Curr Med Res Opin 2016;32:1121-30.

8. Polterauer S, Hefler L, Seebacher V, et al. The impact of lymph node density on survival of cervical cancer patients. Br J Cancer 2010;103:613-6.

9. Polterauer S, Khalil S, Zivanovic O, et al. Prognostic value of lymph node ratio and clinicopathologic parameters in patients diagnosed with stage IIIC endometrial cancer. Obstet Gynecol 2012;119:1210-8.

10. Ataseven B, Grimm C, Harter P, et al. Prognostic value of lymph node ratio in patients with advanced epithelial ovarian cancer. Gynecol Oncol 2014;135:435-40.

11. Ataseven B, Kummel S, Weikel W, et al. Additional prognostic value of lymph node ratio over $\mathrm{pN}$ staging in different breast cancer subtypes based on the results of 1,656 patients. Arch Gynecol Obstet 2015;291:1153-66.

12. He Z, Wu S, Li Q, et al. Use of the metastatic lymph node ratio to evaluate the prognosis of esophageal cancer patients with node metastasis following radical esophagectomy. Plos One 2013;8:e73446.

13. Lee SY, Hwang I, Park YS, et al. Metastatic lymph node ratio in advanced gastric carcinoma: a better prognostic factor than number of metastatic lymph nodes? Int J Oncol 2010;36:1461-7.

14. Li Destri G, Barchitta M, Pesce A, et al. Predictive Value of the Number of Harvested Lymph Nodes and Cut-Off for Lymph Node Ratio in the Prognosis of Stage II and III Colorectal Cancer Patients. J Invest Surg 2019;32:1-7.

15. Iacoponi S, Zapardiel I. European Nationality Influence in the Management of Squamous Cell Vulvar Cancer. Gynecol Obstet Invest 2017;82:349-54.

16. Polterauer S, Schwameis R, Grimm C, et al. Lymph node ratio in inguinal lymphadenectomy for squamous cell vulvar cancer: Results from the AGO-CaRE-1 study. Gynecol Oncol 2019;153:286-91.

17. Wu SG, Zhang WW, Li FY, et al. Lymph node ratio has prognostic value related to the number of positive lymph nodes in patients with vulvar cancer. Future Oncol 2018;14:2343-51.

18. Rauh-Hain JA, Clemmer J, Clark RM, et al. Racial disparities and changes in clinical characteristics and survival for vulvar cancer over time. Am J Obstet Gynecol 2013;209:468.e1-10.

19. Wu SG, Lin QJ, Li FY, et al. Widowed status increases the risk of death in vulvar cancer. Future Oncol 2018;14:2589-98.

20. Polterauer S, Schwameis R, Grimm C, et al. Prognostic value of lymph node ratio and number of positive inguinal nodes in patients with vulvar cancer. Gynecol Oncol 2017;147:92-7.

21. Giulia Mantovani, Fragomeni SM, Inzani F, et al. Molecular pathways in vulvar squamous cell carcinoma: implications for target therapeutic strategies. J Cancer Res Clin Oncol 2020;146:1647-58.

22. Liang W, Zhang L, Jiang G, et al. Development and validation of a nomogram for predicting survival in patients with resected non-small-cell lung cancer. J Clin Oncol 2015;33:861-9.

23. Wang Y, Li J, Xia Y, et al. Prognostic Nomogram for Intrahepatic Cholangiocarcinoma After Partial Hepatectomy. J Clin Oncol 2013;31:1188-95.

24. Kunos C, Simpkins F, Gibbons H, et al. Radiation therapy compared with pelvic node resection for node-positive vulvar cancer: a randomized controlled trial. Obstet Gynecol 2009;114:537-46.

25. Callegaro D, Miceli R, Bonvalot S, et al. Development and 
external validation of two nomograms to predict overall survival and occurrence of distant metastases in adults after surgical resection of localised soft-tissue sarcomas of the extremities: a retrospective analysis. Lancet Oncol 2016;17:671-80.

26. Rose PG, Java J, Whitney CW, et al. Nomograms Predicting Progression-Free Survival, Overall Survival, and Pelvic Recurrence in Locally Advanced Cervical Cancer Developed from an Analysis of Identifiable Prognostic Factors in Patients from NRG Oncology/ Gynecologic Oncology Group Randomized Trials of

Cite this article as: Lei L, Tan L, Zhao X, Zeng F, Xu D. A prognostic nomogram based on lymph node ratio for postoperative vulvar squamous cell carcinoma from the Surveillance, Epidemiology, and End Results database: a retrospective cohort study. Ann Transl Med 2020;8(21):1382. doi: 10.21037/atm-20-3240
Chemoradiotherapy. J Clin Oncol 2015;33:2136-42.

27. Woelber L, Mahner S, Voelker K, et al. Clinicopathological prognostic factors and patterns of recurrence in vulvar cancer. Anticancer Res 2009;29:545-52.

28. Hami LT, Lampe B, Mallmann P, et al. The Impact of Age on the Prognosis of Vulvar Cancer. Oncol Res Treat 2018;41:520-4.

29. Vickers AJ, Cronin AM. Everything you always wanted to know about evaluating prediction models (but were too afraid to ask). Urology 2010;76:1298-301. 